\title{
Interactions of Leukocyte Integrins with Intercellular Adhesion Molecule 1 in the Production of Inflammatory Vascular Injury In Vivo
}

\author{
The Shwartzman Reaction Revisited
}

Lawrence W. Argenbright and Randall W. Barton

Department of Pharmacology, Boehringer Ingelheim Pharmaceuticals Inc., Ridgefield, Connecticut 06877

\begin{abstract}
We have investigated the role of leukocyte-endothelial cell interactions in a rabbit model of hemorrhagic vasculitis. Microvascular injury was produced in the skin by intradermal injection of Salmonella typhosa endotoxin followed $20 \mathrm{~h}$ later by intravenous zymosan, which activates complement. Hemorrhagic necrosis develops in the "prepared" skin sites which is characterized by microthrombi, neutrophil aggregation, platelet and fibrin deposition, and massive extravasation of erythrocytes. Hemorrhage in these Shwartzman-like lesions was quantitated by ${ }^{99}$ Tc-labeled autologous erythrocytes. Inhibition of the hemorrhagic response was obtained with $\mathrm{mAb}$ reactive with ICAM-1 as well as $\mathrm{mAb}$ against the leukocyte CD18 when either was administered intravenously just before intravenous zymosan challenge. This observation suggests that an intravascular event occurring in response to complement activation is required for the development of hemorrhagic vasculitis. We hypothesize that agents which successfully prepare the skin for the Shwartzman response after their intradermal injection do so by promoting increased intercellular adhesion molecule 1 (ICAM-1) expression on the vascular endothelium. Activation of complement then induces CD11/CD18 expression on circulating leukocytes thus producing an intravascular CD11/ CD18-ICAM-1 (leukocyte-endothelium) adhesion event. Inhibition of intravascular leukocyte-leukocyte aggregation with mAb against CD11b (Mac-1) showed partial inhibition of hemorrhage, while mAb against CD11a (LFA-1) showed no inhibitory activity. This type of cytokine-primed, neutrophil-dependent vascular damage may be a model of human vasculitic processes where microvascular damage is produced in the absence of immune-complex deposition. (J. Clin. Invest. 1992. 89:259272.) Key words: neutrophil • endothelium • complement activation • endotoxin • intercellular adhesion molecule 1
\end{abstract}

\section{Introduction}

The development of an inflammatory response requires the adhesion of activated neutrophils to vascular endothelium. While the inflammatory response consists of events which tend

Address correspondence to Lawrence W. Argenbright, Department of Pharmacology, Boehringer Ingelheim Pharmaceuticals, Inc., 900 Ridgebury Rd., P.O. Box 368, Ridgefield, CT 06877.

Received for publication 22 January 1991 and in revised form 29 August 1991.

J. Clin. Invest.

(C) The American Society for Clinical Investigation, Inc.

$0021-9738 / 92 / 01 / 0259 / 14 \$ \$ 2.00$

Volume 89, January 1992, 259-272 to localize and rid the host of invading pathogens, it is not uncommon to find that the same inflammatory response is responsible for significant host tissue damage resulting in morbidity and mortality. The adherence of leukocytes to vascular endothelium represents one of the functional consequences of the expression of a family of leukocyte surface glycoproteins. These adhesion molecules, referred to as the CD18 family of integrins, are heterodimers consisting of distinct alpha subunits: CD11a, CD11b, and CD11c, which associate with a common beta (CD18) subunit. CD11a/CD18 is also called LFA-1, CD $11 \mathrm{~b} / \mathrm{CD} 18$ is called Mac-1, and CD11c/CD18 is also known as p150,95 (1). Stimulation of leukocytes by chemotactic factors including C5a, fMLP, and leukotriene $\mathrm{B}_{4}$ results in rapid expression $(<1 \mathrm{~min})$ of these preformed receptor molecules on the plasma membrane (2-4) as well as increased functional activity of previously expressed adhesion molecules $(5,6)$.

Recent studies have implicated intercellular adhesion molecule-1 (ICAM-1) $)^{1}$ as at least one ligand for the CD18 family of leukocyte integrins $(1,7)$. ICAM-1 expression is normally present at low levels on several nonhematopoietic cell types, but can be upregulated by a variety of cytokines. Stimulation of endothelial cells by interleukin 1, tumor necrosis factor, interferon $\gamma$, as well as bacterial endotoxins, greatly increases ICAM-1 expression by these cells $(8,9)$. The significance of CD18/ICAM-1 interaction in contributing to certain inflammatory responses in vivo is clear (1).

Proper regulation of neutrophil adhesion is critical to host defense. Over the past several years, a group of pediatric patients has been described which is genetically deficient in the CD18 adhesion glycoproteins $(10,11)$. Neutrophils from these patients are defective in adhesion related functions such as aggregation, adhesion to endothelium, and spreading on surfaces. That most of these patients have died before the age of two years because of chronic bacterial infections illustrates the critical role that neutrophil adhesion plays in homeostasis.

Microvascular damage as seen in autoimmune diseases such as systemic lupus erythematosus (SLE) has often been considered as resulting from deposition of immune complexes in the blood vessel wall. The Arthus reaction (intradermal injection of antigen in a previously sensitized animal) has become a model of immune complex-mediated vascular damage. Recently, Philips et al. suggested that an alternative mechanism of vascular damage may occur in SLE involving intravascular activation and aggregation of neutrophils in the absence of immune complex deposition (12), and that the Shwartzman reaction may be a model of this type of vasculitis not mediated by immune complex deposition.

1. Abbreviation used in this paper: ICAM-1, intercellular adhesion molecule 1 . 

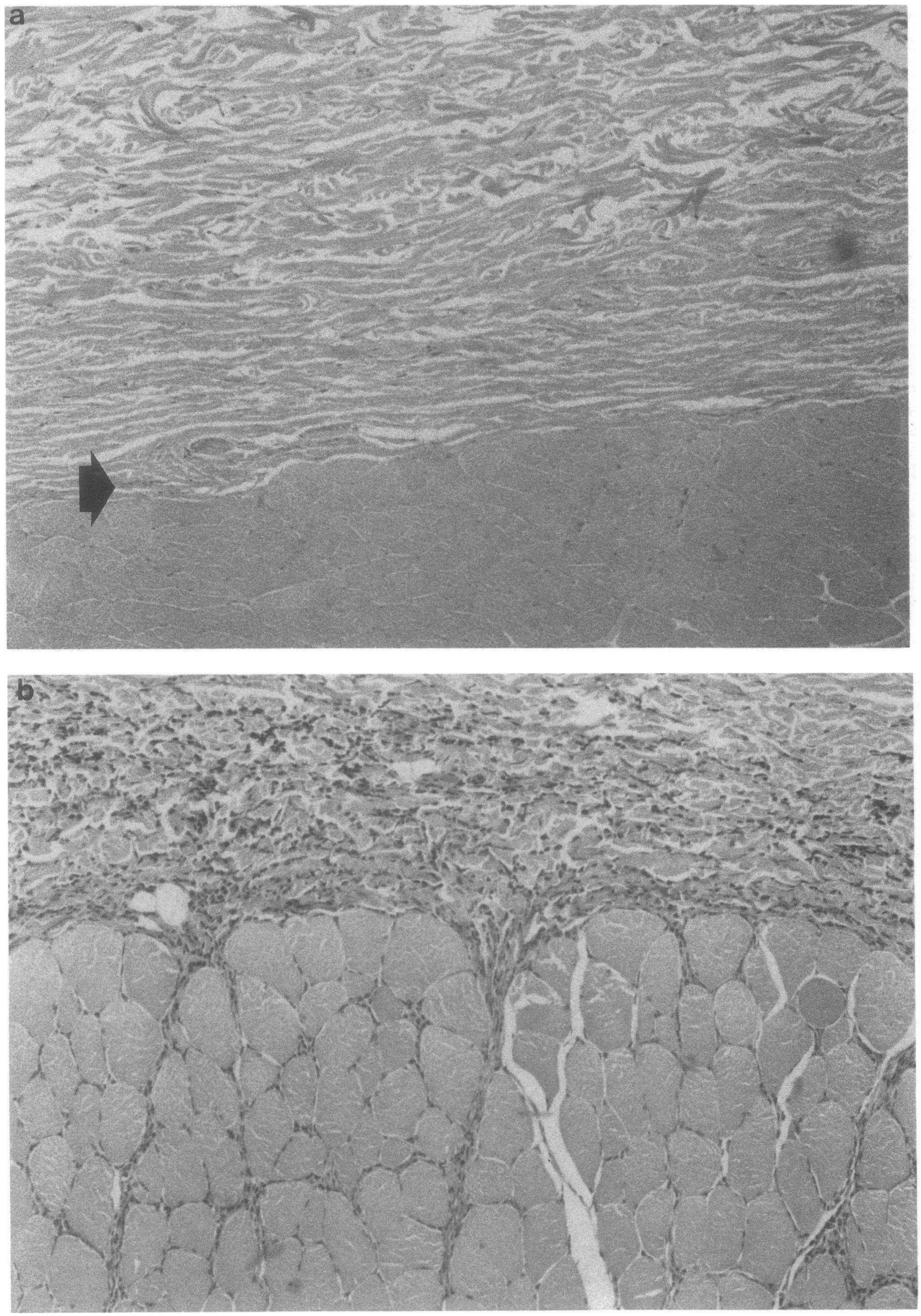


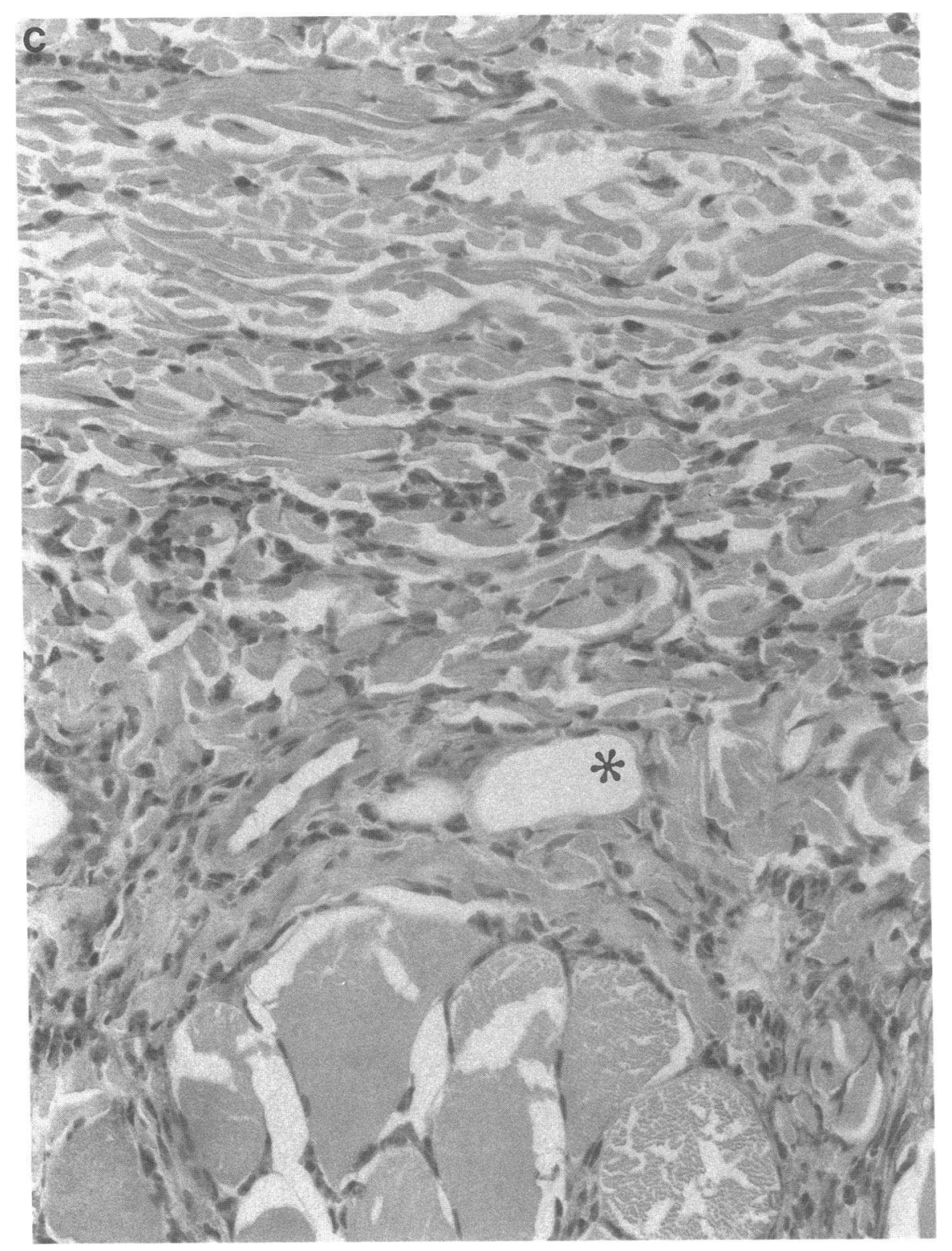

Figure 1. (a) Histologic section of rabbit skin illustrating the junction between the dermis and the paniculus carnosus muscle (arrow), normal, $\times 200$. (b) Rabbit skin $20 \mathrm{~h}$ after i.d. injection of $30 \mu \mathrm{g}$ LPS. Inflammatory infiltrate (predominantly neutrophils) is evident in the deep dermis, extending down into the subcutaneous muscle, $\times 200$. (c) Same tissue as in $b$ showing leukocytes in the dermis. The lumen of several vessels is patent and appears normal (asterisk), $\times 300$.
As originally described by Gregory Shwartzman in 1928 (13), intravenous (i.v.) injection of Bacillis typhosus culture filtrate produced hemorrhagic necrosis in skin sites where the same culture filtrate had been injected intradermally $24 \mathrm{~h}$ previously. In spite of much study over the past 60 years, the mechanism of vascular damage in the Shwartzman reaction is still not clear. It is now generally accepted that endotoxin was the active moiety in Shwartzman's culture filtrates; the same response can be elicited by injecting endotoxin rather than culture filtrate (14). Whereas a number of agents can substitute as the i.v. "challenge", it is more difficult to substitute for endotoxin as a "preparative" agent. How a tissue is prepared for Shwartzmanlike lesions is a question which we have attempted to answer.

In this manuscript we report that monoclonal antibodies against ICAM-1, CD18, or CD11b, but not CD11a, can block the hemorrhagic vasculitis occurring in Shwartzman lesions, suggesting that preparation may require upregulation of ICAM-1 on vascular endothelium. Further, the response to the intravascular challenge appears to be an intravascular event dependent on both ICAM-1 and CD11b (Mac-1) activity.

\section{Methods}

Male, New Zealand White rabbits, weighing from 2.8 to $3.8 \mathrm{~kg}$, were used throughout these studies. They had access to commercial rabbit chow and tap water ad lib.

Experimental protocol. The hair on the dorsum was removed with electric clippers. Lipopolysaccharide W (Salmonella typhosa 0901; Difco Laboratories, Detroit, MI), in sterile $0.9 \%$ saline was injected i.d. in $0.2-\mathrm{ml}$ vol. Each animal received six replicate preparative injections of $0,3,10,30$, and $100 \mu \mathrm{g}$ LPS. 18-20 h later, in vivo labeling of red blood cells was performed by injecting $20 \mu \mathrm{g} / \mathrm{kg}$ stannous chloride (PYROLITE diagnostic kit, for ${ }^{99 \mathrm{~m}} \mathrm{Tc}$ labeling of human blood; E. I. duPont, Billerica, MA), followed $30 \mathrm{~min}$ later by $200 \mu \mathrm{Ci} / \mathrm{kg}{ }^{99 \mathrm{~m}} \mathrm{Tc}$ (sodium pertechnetate; Syncor Int'l Corp., Stamford, CT). This procedure results in $>97 \%$ of the ${ }^{99 m} \mathrm{Tc}$ activity in whole blood being associated with RBC's. After ${ }^{99 \mathrm{~m}} \mathrm{Tc}$ injection, zymosan A (Sigma Chemical Co., St. Louis, MO), in sterile saline, as a challenging agent, was injected via a marginal ear vein at $10 \mathrm{mg} / \mathrm{kg}$. 4-6 h later, a sample of blood was drawn from each animal for calculation of ${ }^{99 \mathrm{~m}} \mathrm{Tc}$ labeling efficiency and specific activity. Euthanasia was performed by sodium pentobarbital overdose.

The dorsal skin of each animal, containing all sites of i.d. injection, 


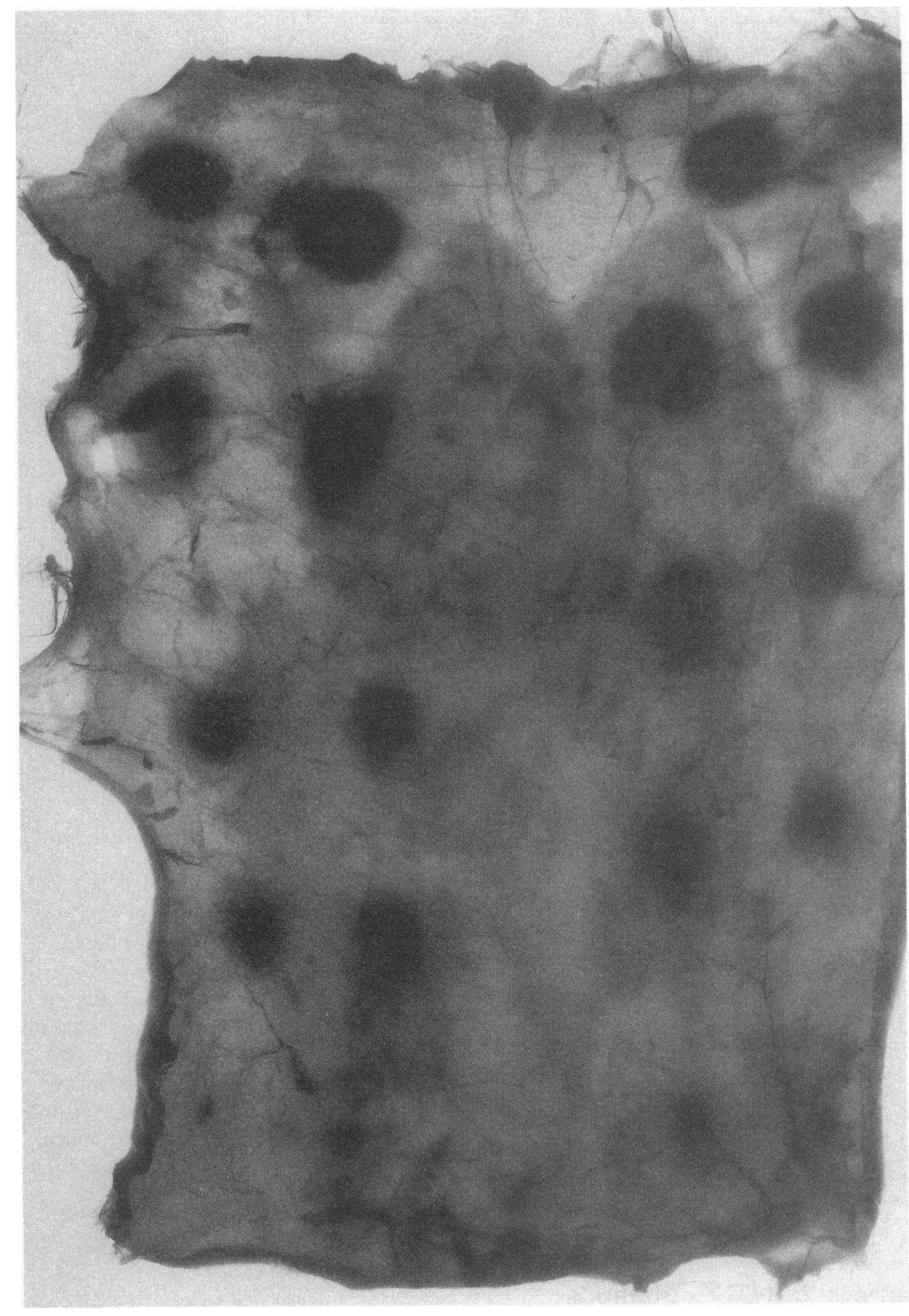

Figure 2. Shwartzman lesions in rabbit skin. Illustrated are groups of four replicate injections of (top to bottom) 100,30,10,3, and 1 $\mu \mathrm{g}$ LPS per site. $20 \mathrm{~h}$ after LPS injections, complement was activated by i.v. zymosan. 6 $\mathrm{h}$ later, the skin was removed and the hemorrhagic lesions were photographed from the dermal side. was removed with a scalpel, laid out flat and frozen under a Pyrex dish containing solid $\mathrm{CO}_{2}$ and methanol. When frozen, injection sites were removed with a specially made skin punch which fit into an electric drill, thus allowing removal of skin pieces of reproducible size. The ${ }^{99 m} \mathrm{Tc}$ activity in each skin sample was then determined in an autogamma counter (Model 5870 [window setting of 120-160 kiloelectron volts]; Packard Instrument Co., Inc., Meriden, CT). The ${ }^{99 \mathrm{~m}} \mathrm{Tc}$ activity in each sample was converted to blood equivalents by dividing by the specific activity $\left({ }^{99 m} \mathrm{Tc} \mathrm{cpm} / \mathrm{known}\right.$ blood volume) of the blood from that animal.

Shwartzman-like lesions were produced in several additional animals, not labeled with ${ }^{99 \mathrm{~m}} \mathrm{Tc}$, and skin lesions were prepared for routine histological evaluation.

Antibody treatment. The mouse monoclonal antibody R6.5 (IgG2a, anti-human ICAM-1) has been described previously (15). R15.7, also a mouse monoclonal (IgG1) was raised against the canine CD18 adhesion glycoprotein. Its specificity was demonstrated by its ability to in- hibit homotypic aggregation of JY cells (16), and by its binding to mouse $\times$ human hybrids (17) expressing mouse CD11/human CD18 but not to hybrids expressing human CD11/mouse CD18. R7.1 (mouse IgGI, antiCD1 la) binds to rabbit leukocytes as ascertained by flow cytometry. LM2 (mouse IgG1, antiCD11b) has been described previously (18). Purified mouse IgG was used as control. All antibodies, as purified protein, were dissolved in sterile saline and injected i.v. at $2 \mathrm{mg} / \mathrm{kg}$.

Immunohistochemical demonstration of ICAM-1. In one additional animal, preparative injections of LPS were made as usual. $20 \mathrm{~h}$ later, biopsies of PBS- and LPS-injected skin were fixed and stained following the protocol of Gu and McGrath (19). Briefly, samples were fixed in $4 \%$ paraformaldehyde, embedded in paraffin, cut in $5-\mu \mathrm{m} \mathrm{sec}-$ tions, dewaxed, and incubated with either mAb R6.5 or control mouse IgG. Detection of bound antibody was performed using biotinylated goat anti-mouse, peroxidase conjugated streptavidin and AEC substrate (BioGenex Laboratories, San Ramon, CA). 
Upregulation of CD11/CD18 in vivo. In one experimental animal, a blood sample was obtained before and $30 \mathrm{~min}$ after the i.v. zymosan challenge. Blood cells were washed free of plasma in Dulbecco's PBS containing $0.13 \% \mathrm{NaN}_{3}$ (DPBS-Z). Aliquots of $100 \mu \mathrm{l}$ of blood cell suspension were pelleted and resuspended in $25 \mu$ l of the mAb's R15.7 (anti-CD18), LM2 (antiCD11a), or control mouse IgG. The cells were incubated for $20 \mathrm{~min}$ at $4^{\circ} \mathrm{C}$, washed $2 \times$ with DPBS-Z, and incubated with $25 \mu \mathrm{l}$ of a 1:10 dilution of affinity purified, FITC-conjugated $(\mathrm{ab})_{2}^{\prime}$ goat anti-mouse IgG (heavy and light chain specific) (Cappel Research Products, Durham, NC) at $4^{\circ} \mathrm{C}$ for $20 \mathrm{~min}$. The cells were then washed, treated with FACS ${ }^{\otimes}$ lysing solution (Becton Dickinson Immunochemistry Products, San Jose, CA) to lyse erythrocytes, and fixed in 1\% paraformaldehyde. Analysis was performed using a FACS ${ }^{\circledR} 440$ (Becton Dickinson).

\section{Results}

$18-20 \mathrm{~h}$ after the intradermal injection of $S$. typhosa endotoxin into rabbit skin, an accumulation of leukocytes (mostly neutrophils, some monocytes) was seen, with the strongest reaction occurring in the deep dermis just above the panniculus carnosus muscle (Fig. 1). In general, the accumulation of leukocytes had ceased by $18 \mathrm{~h}$ since dermal vessels were mostly free of adherent leukocytes and no evidence of microthrombi was seen. When animals were then challenged by systemic activation of complement, only the prepared intradermal sites became grossly hemorrhagic over a 4-6-h period (Fig. 2). Histologically, the response to challenge consisted of intravascular aggregation of leukocytes (Fig. 3), fibrin deposition, and massive extravasation of RBC's which was superimposed on the leukocyte accumulation induced by the endotoxin preparation. Quantitation of hemorrhage with autologous ${ }^{99 \mathrm{~m}} \mathrm{Tc}-\mathrm{RBC}$ showed an endotoxin dose-dependent response (Fig. 4). When $100 \mu \mathrm{l}$ of zymosan-activated rabbit plasma was substituted for endotoxin as the preparative injection, the hemorrhagic response after challenge was not significantly different from that of PBS-injected sites $(14 \pm 4,21 \pm 5$, and $53 \pm 6 \mu \mathrm{l} \mathrm{blood/site} \mathrm{for}$ PBS-, zymosan-activated plasma-, and LPS-injected sites, respectively).

To evaluate the role of ICAM- 1 and the leukocyte integrins in the development of these hemorrhagic lesions, animals were pretreated with appropriate mAb. Administration of either antiCD18 or antilCAM-1 before both i.d. preparative and i.v. systemic challenge injections produced strong inhibition of the hemorrhagic response (Fig. 5). While that protocol showed that the hemorrhagic response was dependent on both CD18 and ICAM-1, it was not known whether these antibodies blocked a response to the preparative injection, or blocked some aspect of the response occurring after systemic complement activation. We therefore modified the protocol to administer $\mathrm{mAb}$ only before systemic challenge. Fig. 6 shows that strong $(>80 \%)$ inhibition of hemorrhage was obtained when either the CD18 or ICAM-1 molecule was occupied before complement activation. While $\mathrm{mAb}$ recognizing $\mathrm{CD} 1 \mathrm{lb}$ (Mac-1) produced signifcant inhibition (62\%), mAb against CD11a (LFA-1) was without effect.

Fig. 7 photographically illustrates ICAM-1 expression on dermal vessels $20 \mathrm{~h}$ after the i.d. preparative injection of LPS. Such expression was seen on most venous vessels in the inflammatory sites, whereas ICAM-1 expression was only occasionally seen in PBS-injected skin, and with what appeared to be less intense staining.
Leukocytes were activated by i.v. zymosan (systemic activation of complement) as evidenced by increased binding of mAb reactive with either CD18, or CD11a (Mac-1). Fig. $8 a$ illustrates that a significant basal CD18 expression increased by threefold. Fig. $8 b$ shows that little binding of anti-CD11a occurred until after zymosan injection.

While intravascular adhesion of leukocytes to endothelium consistently occurred after i.v. challenge, the endothelium of animals pretreated with $\mathrm{mAb}$ reactive with $\mathrm{CD} 18$ was relatively free of adherent leukocytes (Fig. 9). Pretreatment with antiCD $1 \mathrm{lb}$ did not inhibit leukocyte-endothelial adhesion, but intravascular leukocyte-leukocyte aggregation was not seen (Fig. 11).

\section{Discussion}

When complement is activated by i.v. zymosan $20 \mathrm{~h}$ after i.d. injection of endotoxin, hemorrhagic vasculitis ensues in those prepared skin sites. These Shwartzman-like lesions are characterized histologically by microthrombi, neutrophil aggregation, platelet and fibrin deposition, and later, massive extravasation of RBC. The requirement of the local preparatory injection is absolute; microvascular damage of this sort is not produced simply by activating complement. No hemorrhage in normal or vehicle injected skin was detected after challenge.

It has been noted by several investigators $(13,14)$ that thrombosis and hemorrhage only follow challenge in i.d. sites where erythematous, inflammatory reactions developed in response to preparation. Several groups have shown $(14,20)$ that accumulation of ${ }^{51} \mathrm{Cr}$-leukocytes peaks 3-4 $\mathrm{h}$ after the preparative injections, and that there was a direct, quantitative relationship between accumulation of leukocytes after preparation and the ensuing hemorrhage following challenge. Shwartzmanlike lesions don't develop in neutropenic animals (21), injection of neutrophil-derived proteases produces vascular damage which, ultrastructurally, resembles Shwartzman lesions (22), and inhibition of leukocyte proteases effectively inhibits this hemorrhagic response (23). These observations imply a requirement for neutrophil accumulation in local areas after exposure to a suitable preparative agent.

Our data suggest a different neutrophil dependence than protease release from accumulated leukocytes. When leukocytes were allowed to accumulate in the skin after i.d. endotoxin, and then mAb directed against the CD18 leukocyte adhesion glycoprotein was administered as a single injection just before i.v. challenge, hemorrhagic lesions were dramatically inhibited. This observation suggests that leukocytes accumulated in the interstitium are not responsible for the ensuing hemorrhage, but that the intravascular leukocyte aggregation (Fig. 3) is what leads to the hemorrhagic response. Products of complement activation, 'as well as leukotrienes and bacterial chemotactic peptides activate neutrophils and stimulate, within minutes, the expression of preformed CD18 complex on the leukocyte plasma membrane. We have now demonstrated that this upregulation occurs after the challenge injection in these experiments. This suggests, therefore, that the activation of circulating leukocytes results in a CD18-dependent leukocyte-endothelial cell adhesion which is a necessary event for the development of Shwartzman-like lesions. Fig. 9 shows a representative dermal venule from an animal pretreated with an antiCD18 mAb (R15.7) just before challenge. Although a 
a

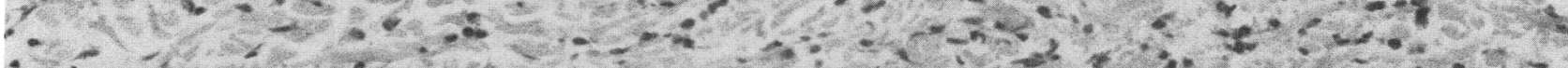

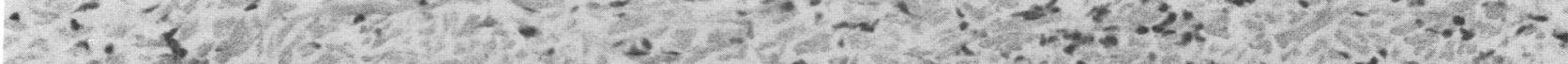

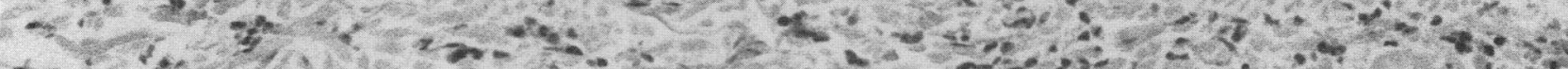

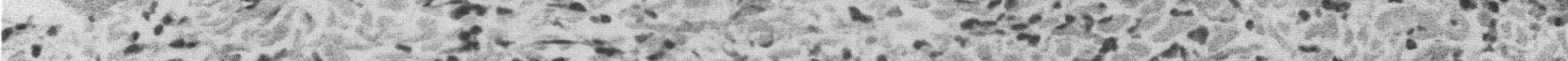

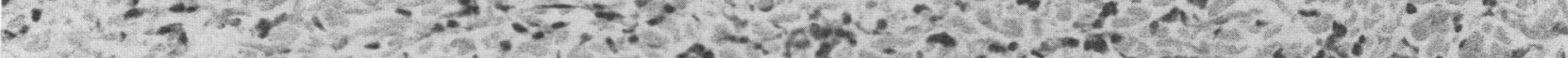
I. 5 -

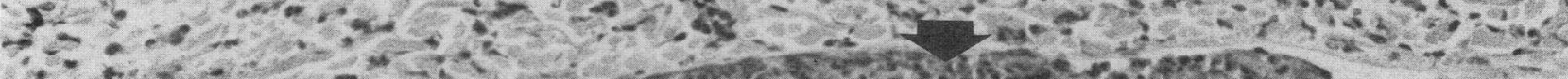

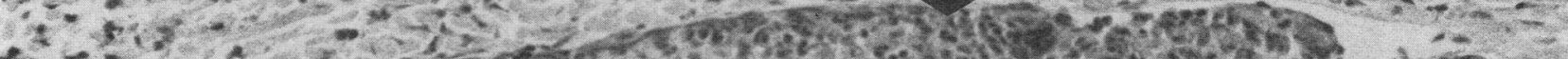

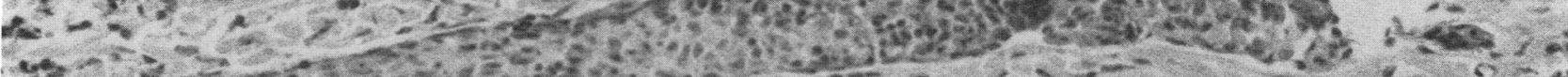

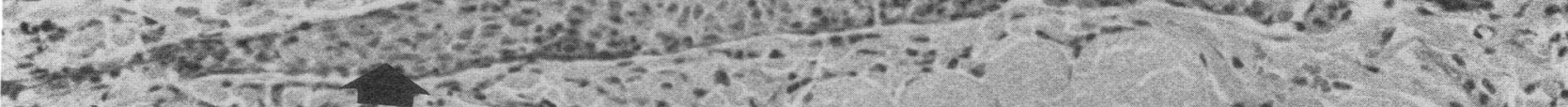
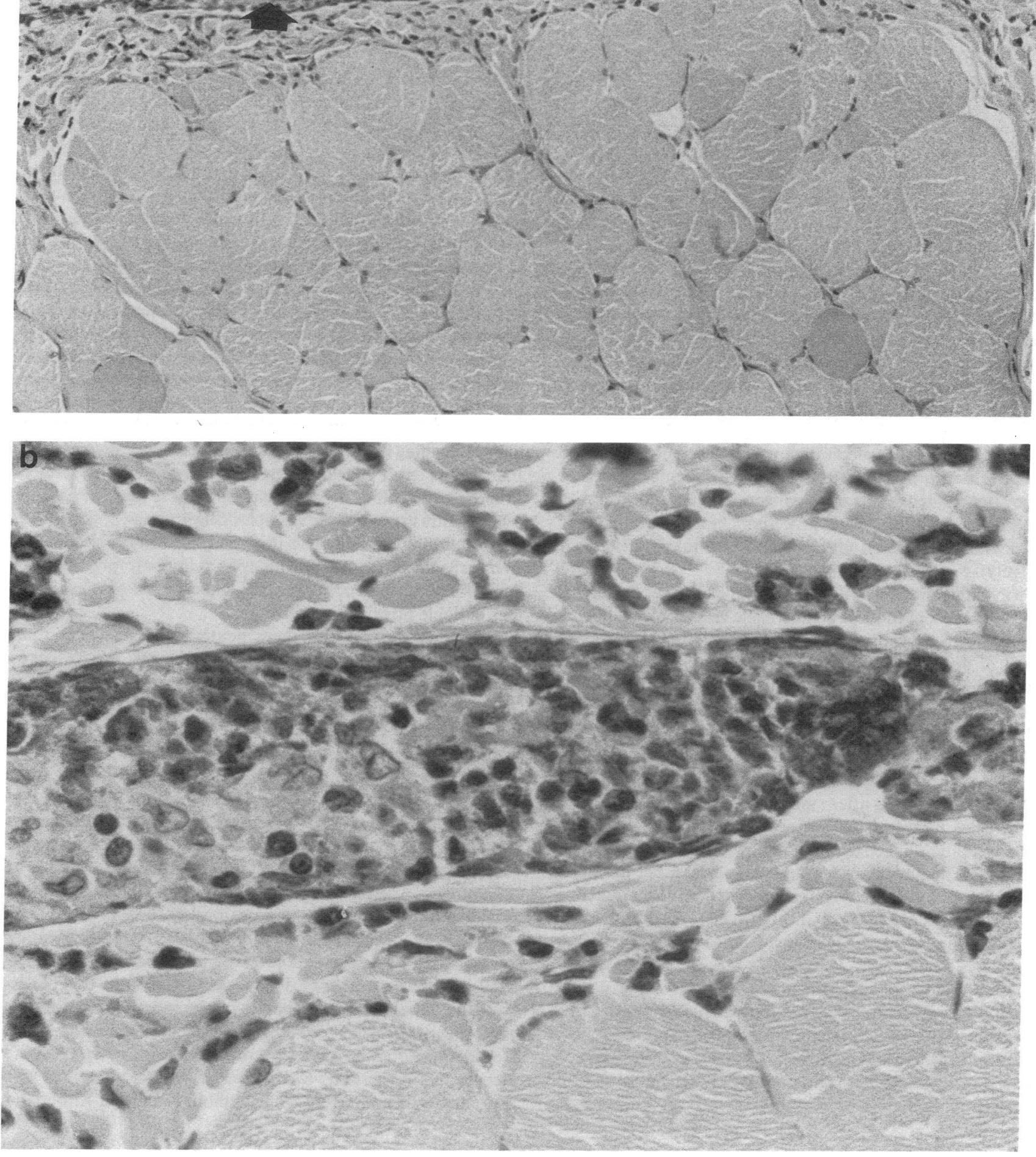


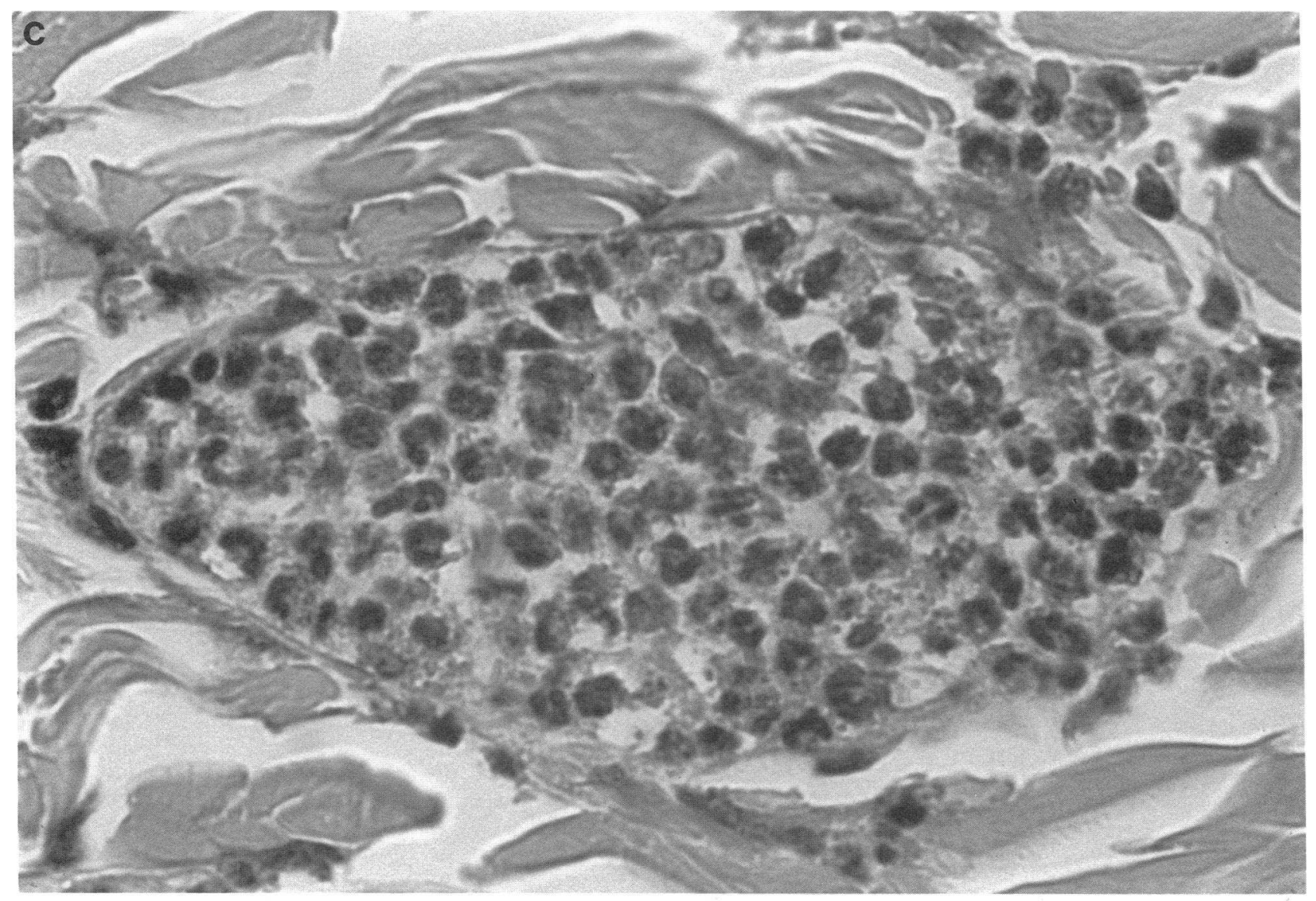

Figure 3. (a) and (b) Photomicrograph of rabbit skin, previously injected with $30 \mu \mathrm{g} \mathrm{LPS,} 2 \mathrm{~h}$ after zymosan challenge. Superimposed on the leukocyte infiltration resulting from the LPS preparative injection (see Fig. $1 \mathrm{~b}$ ), is seen intravascular leukocyte aggregation and thrombus formation (arrows), $\times 300$ and $\times 500(c)$. Another vessel from the same lesion, note intravascular plugging by leukocyte aggregate, $\times 500$.

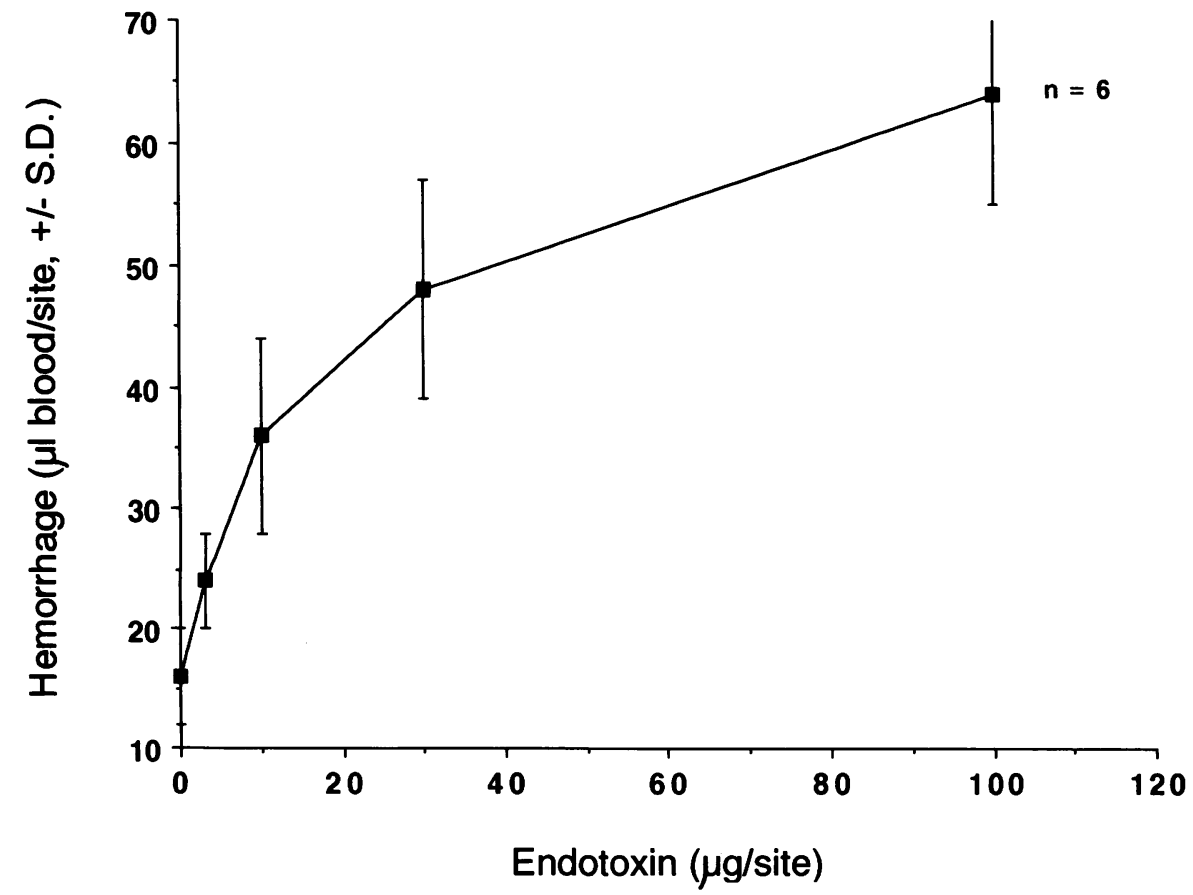

Figure 4. Quantitation of hemorrhage in Shwartzman lesions in rabbit skin. RBC's were labeled in vivo with ${ }^{99 \mathrm{~m}} \mathrm{Tc}$. Intradermal injection of LPS was performed $20 \mathrm{~h}$ before i.v. injection of zymosan. $6 \mathrm{~h}$ after zymosan, hemorrhagic lesions (see Fig. 2) had developed. These lesions were removed, and ${ }^{99 m} \mathrm{Tc}$ activity in each was determined. It can be seen that a LPS dosedependent hemorrhage has been produced. 


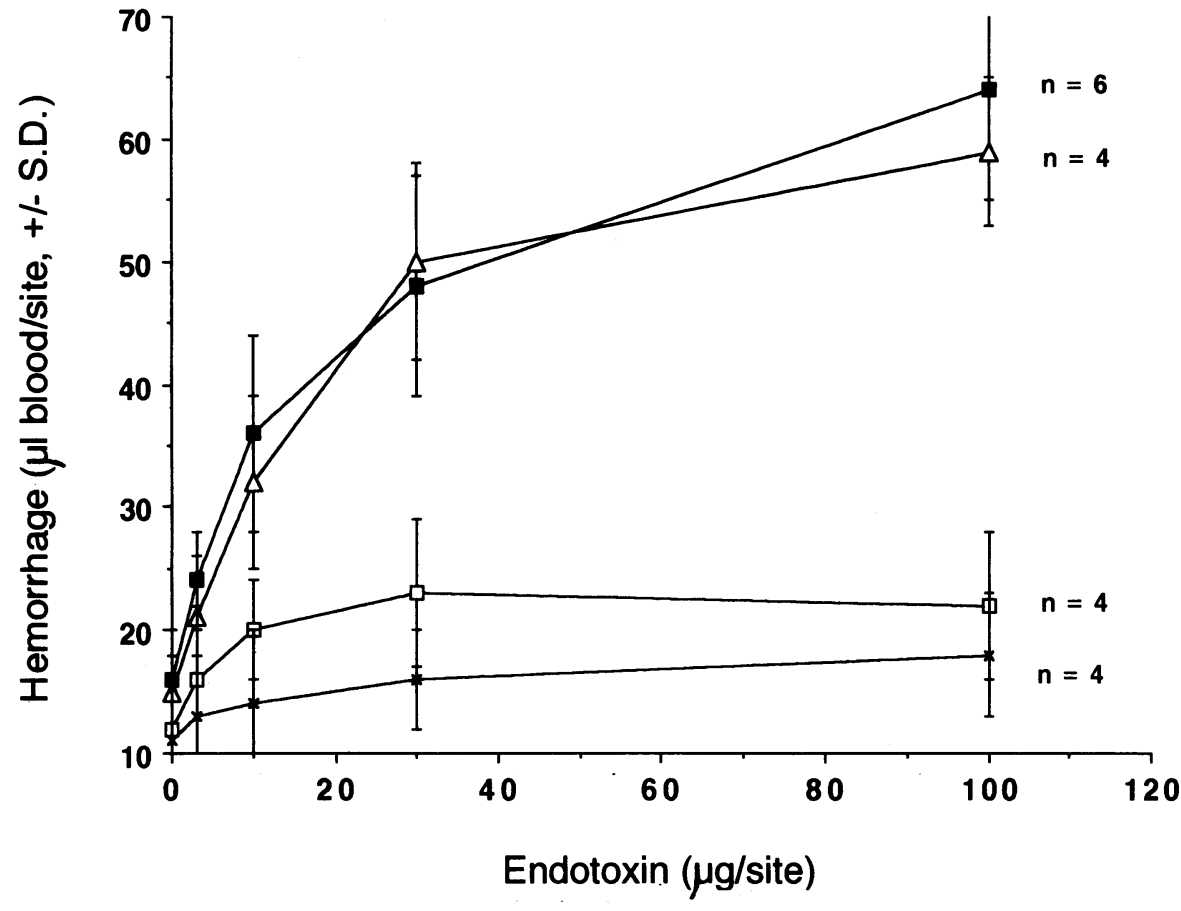

Figure 5. Shwartzman-induced hemorrhage in rabbit skin. Pretreatment of animals with mAb reactive with either $\mathrm{CD} 18(\mathrm{R} 15.7, \mathrm{X})$ or with ICAM-1 $(\mathrm{R} 6.5, \square)$ both before the i.d. preparative LPS injections and again just before the intravenous zymosan injection. Significant inhibition of the hemorrhagic response by either mAb was obtained. Mouse IgG $(\Delta)$ was administered as a control. All antibodies were administered at $2 \mathrm{mg} / \mathrm{kg}$, i.v. few neutrophils are adherent to the endothelium, most of the endothelial surface is free of adherent cells, and leukocyte aggregates as in Fig. 3 were not seen.

If extravasated leukocytes are not producing the vascular damage, what is the nature of the preparative injection? The strong inhibitory effect of an antilCAM-1 mAb suggests that ICAM-1 has been upregulated in areas treated with the preparative agent. Bacteria $(14,24)$, endotoxin $(14,24)$, IL-1 $\beta$ (20, 24), TNF $\alpha$ (24) have been' used successfully as preparative agents for the Shwartzman response. Endotoxin and these cytokines have also been shown to induce ICAM-1 expression on cultured vascular endothelial cells $(8,9,25)$, and our data demonstrate ICAM-1 upregulation in response to LPS in vivo. It is not sufficient to call leukocytes into a tissue to successfully prepare a tissue for the Shwartzman reaction. While agents which can work as preparative agents do produce leukocyte accumulation in vivo, other chemotactic agents, such as IMLP which also produces local infiltration, do not prepare tissues for the Shwartzman response, although they can successfully challenge (26). We have found that i.d. C5à, while producing leukocyte accumulation (Fig. 10), will not support the Shwartzman response, and further, that $\mathrm{C} 5 \mathrm{a}$ did not induce ICAM-1 expression on cultured endothelial cells (Dr. Christina Myers, personal communication). Rampart et al. (27) have shown that IL-1-induced neutrophil accumulation in rabbit skin can be blocked by protein synthesis inhibitors, while neutrophil influx in response to $\mathrm{C} 5 \mathrm{a}$, $\mathrm{MPL}$, and $\mathrm{LTB}_{4}$ was unaffected. We therefore consider that the nature of preparation for Shwartzman-

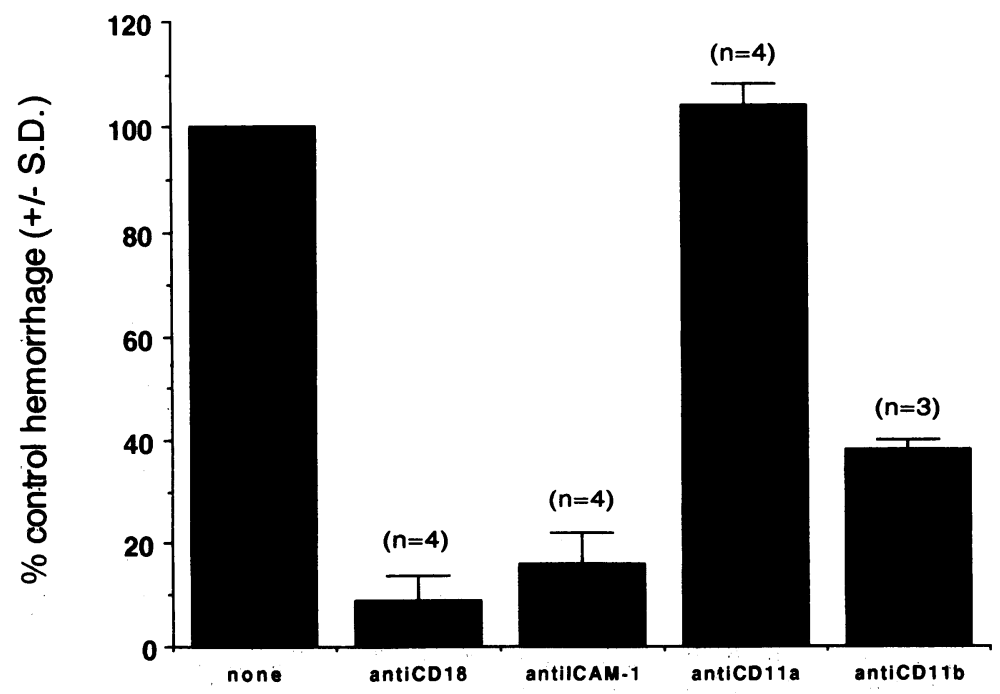

Figure 6. Effect of antiadhesion mAb on Shwartzman-induced hemorrhage in rabbit skin. In animals whose skin had been primed with i.d. LPS injections, mAb reactive with various adhesion molecules were administered i.v. at $2 \mathrm{mg} / \mathrm{kg}$ as a single dose, just before i.v. zymosan challenge. $6 \mathrm{~h}$ after challenge, the hemorrhagic lesions were removed from the skin, and the blood content of each was determined. The graph illustrates the effect of mAb on the 100 $\mu \mathrm{g}$ LPS/site lesions. AntiCD18 and antiICAM-1 were both very effective at blocking the development of hemorrhage. AntiCD1 la was not inhibitory, whereas antiCD11b inhibited hemorrhage by $62 \%$. 

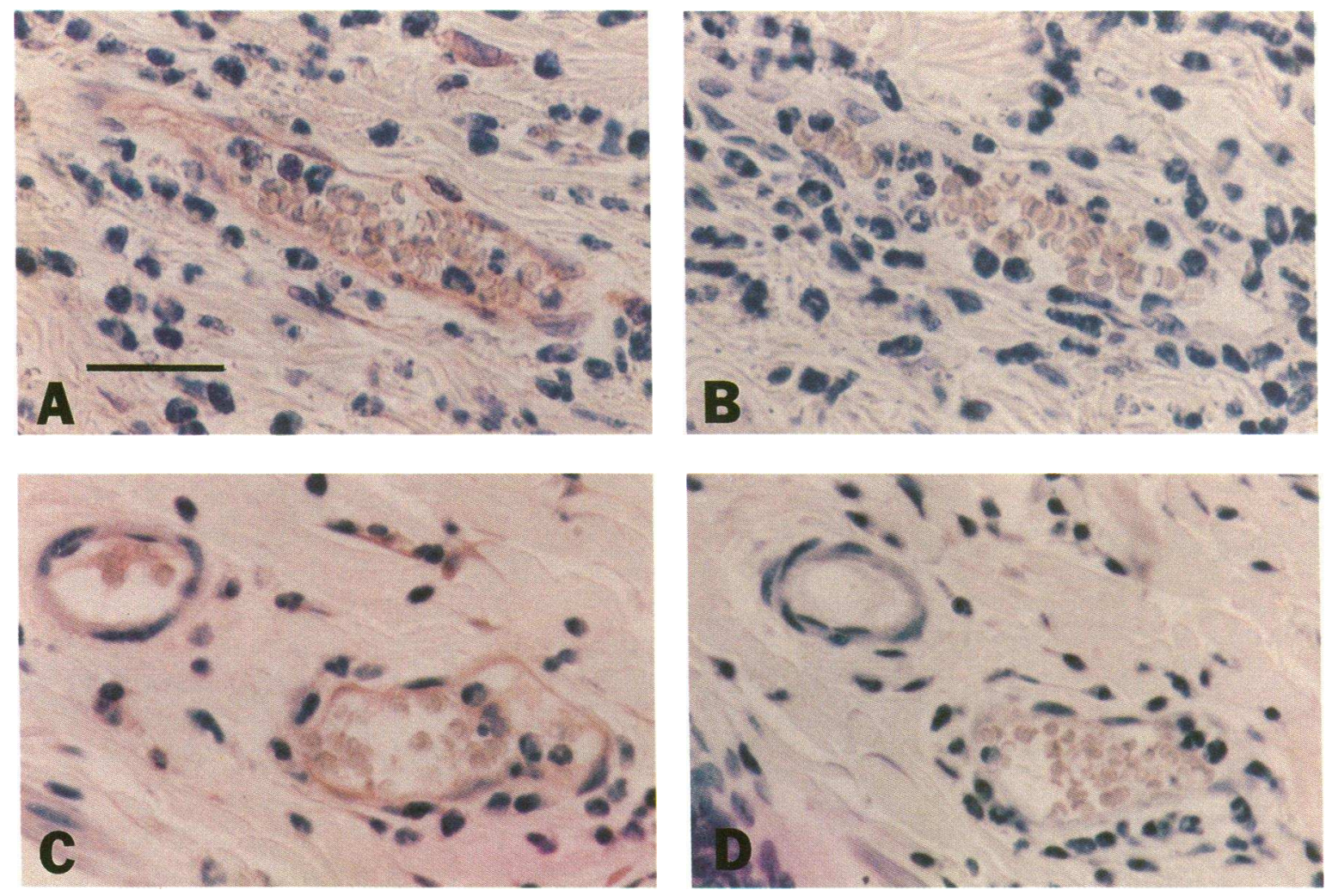

Figure 7. $A$ and $C$ illustrate the immunolocalization of ICAM-1 on rabbit dermal blood vessels $20 \mathrm{~h}$ after the intradermal injection of $3 \mu \mathrm{g} S$. typhosa endotoxin. R6.5 was used as primary mAb. Uniform staining of the endothelium is seen. The majority of vessels in endotoxin treated skin showed this ICAM-1 expression, whereas only an occasional vessel in saline injected skin stained for ICAM-1. $B$ and $D$ show the same vessels as in $A$ and $C$, respectively, where mouse IgG (control) was used as primary mAb. No nonspecific staining is evident. Bar equals $50 \mu \mathrm{m}$.
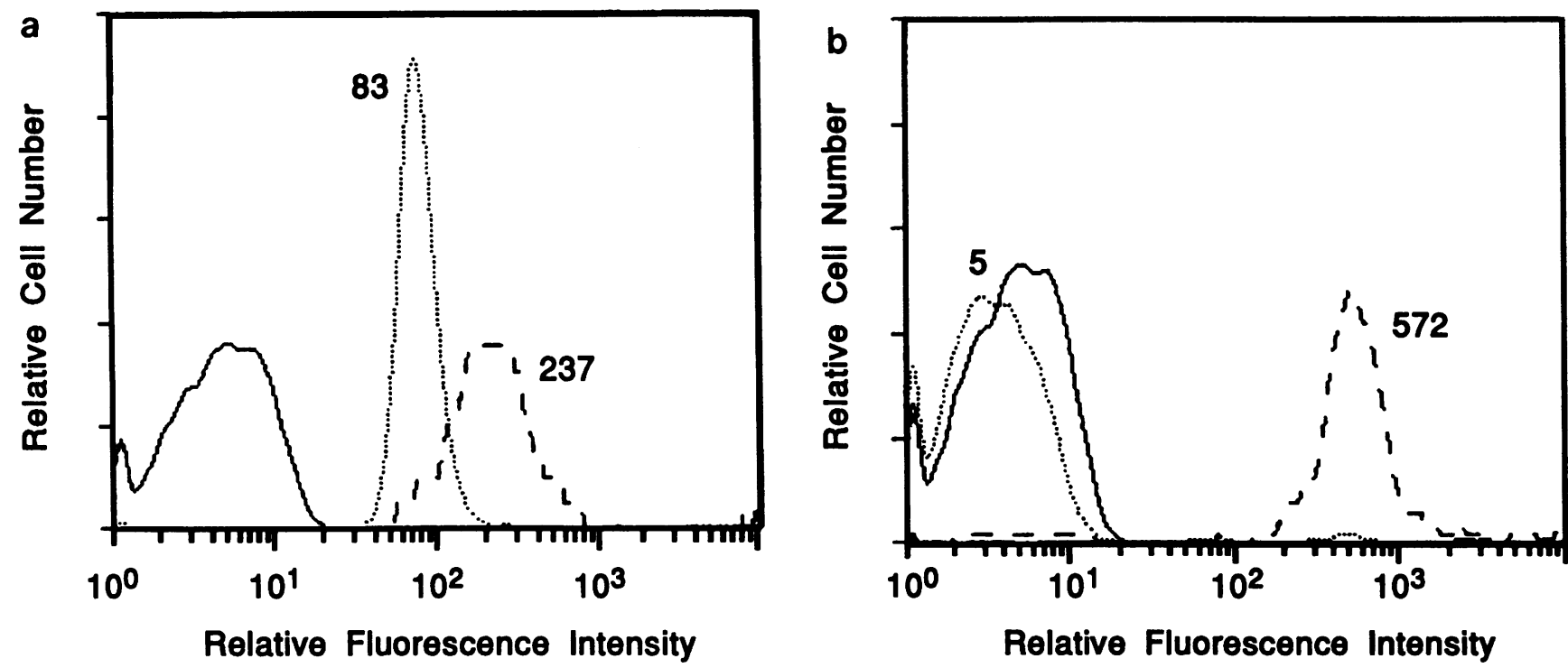

Figure 8. Flow cytometric analysis of rabbit blood before and $30 \mathrm{~min}$ after i.v. injection of zymosan (complement activation). (a) Normal rabbit leukocytes, not incubated with primary antibody, showed a low level of (auto)fluorescence. Significant basal CD18 expression was found on normal circulating leukocytes (mean fluorescence of 83) which increased threefold (mean fluorescence of 237) after challenge. (b) As assessed by mAb LM2, no CD1 lb (Mac-1) was detected on unstimulated leukocytes whereas zymosan challenge stimulated a dramatic upregulation of the CD1 1 b.antigen (mean fluorescence of 572). (Solid line represents normal control leukocytes not incubated with primary antibody.) 


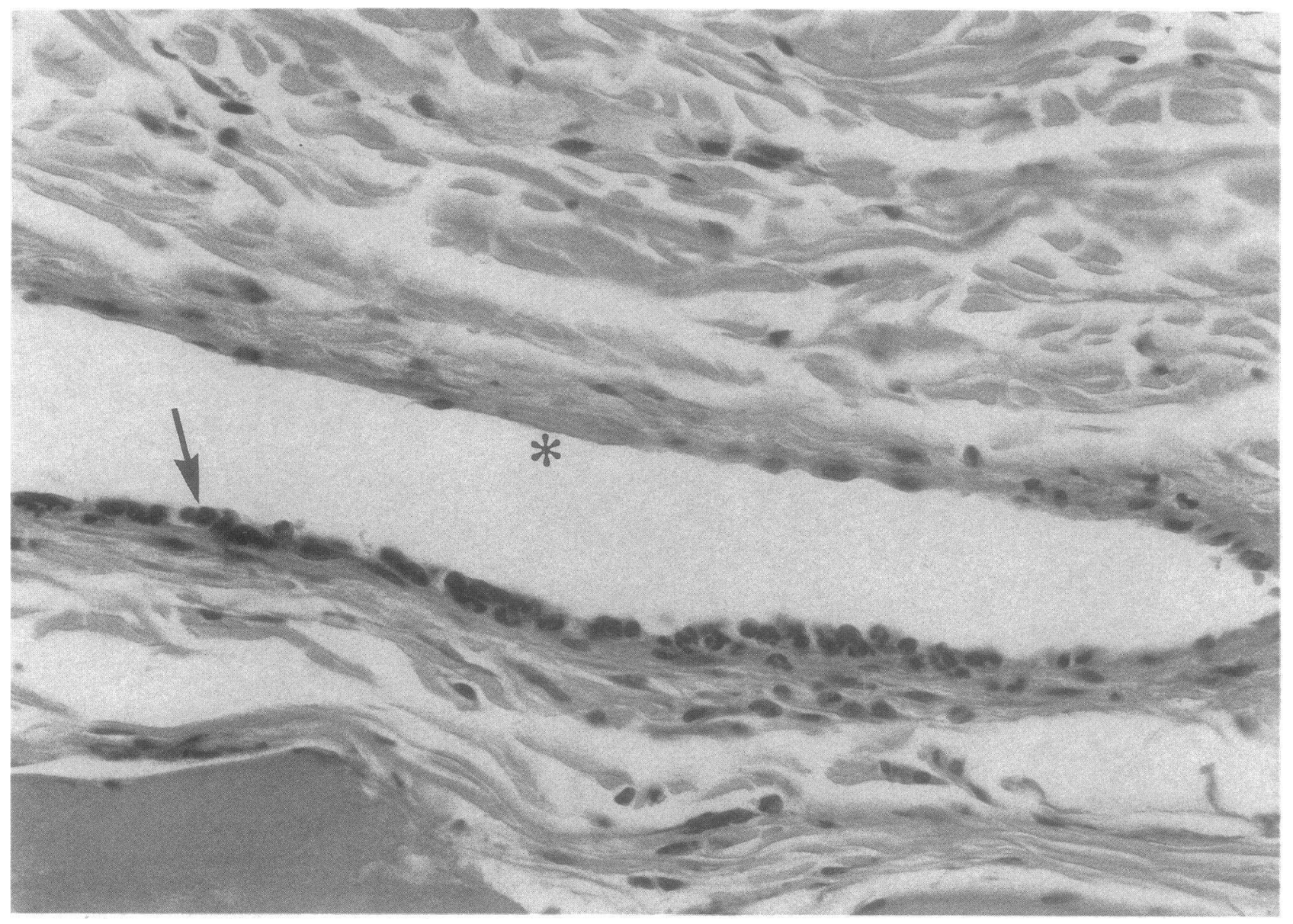

Figure 9. Histology of representative blood vessel from Shwartzman lesion in a rabbit pretreated with a mAb against CD18. Although this section shows some leukocyte-endothelial adhesion (arrow), most of the vessel surface is free of adherent cells (asterisk), and no leukocyte aggregates are seen. Hemorrhage was blocked by $>90 \%$ in these lesions, $\times 300$.

like inflammatory lesions is the upregulation of ICAM-1 on endothelium, and that the accumulated leukocytes in the interstitium after the preparative injection are as much a consequence of ICAM-1 upregulation as is the hemorrhage following challenge.

While these data suggest that a CD18-ICAM-1 dependent leukocyte-endothelial cell adhesion is necessary for this vasculitis to occur, intravascular leukocyte-leukocyte aggregation appears also to be required. Anderson et al. (18) demonstrated that human granulocytes aggregate in response to $\mathrm{C5a}$ in vitro, and that this aggregation was inhibited by $97 \%$ by the same antiCD11b (Mac-1) antibody used in our studies. Antibody recognizing CD1 1a (LFA-1) was not inhibitory. If one contrasts the intravascular aggregation seen in the uninhibited Shwartzman response (Fig. 3) with vessels from an animal pretreated with antiCD11b (Fig. 11), it is clear that while CD18/ ICAM-1 dependent leukocyte adhesion was not completely inhibited as evidenced by a single layer of leukocytes adherent to the vessel surface, no leukocyte aggregation occurred, and hemorrhage was partially inhibited in these animals.

We have previously shown, by intravital microscopic ob- servation of the mesenteric microcirculation in the rabbit, that $\mathrm{mAb}$ against CD1 $1 \mathrm{a}$ and $\mathrm{CD} 1 \mathrm{lb}$ are effective in blocking the initial adhesion of C5a stimulated leukocytes to the vascular endothelium (28). Considering the observation that antiCD1 1a did not inhibit the Shwartzman hemorrhage, these data suggest that the initial CD18/ICAM-1 dependent leukocyte adhesion in the Shwartzman response is mediated by both LFA- 1 and Mac-1. Inhibiting only LFA-1 will not block the hemorrhagic lesions. Inhibiting Mac-1 activity blocks both leukocyte-endothelial cell adhesion as well as leukocyte-leukocyte aggregation. Fig. 12 illustrates the proposed mechanism of the development of this "cytokine primed", intravascular leukocyte-mediated vascular damage.

There may be numerous conditions of human pathology which have as a basis events similar to those presented by this model. Complement-mediated neutrophil aggregation has been suggested as contributing to the tissue damage in various disease entities including adult respiratory distress syndrome (29), pancreatitis (30), and myocardial infarction (31). The multiple vascular thrombosis and tissue necrosis of the Shwartzman reaction has also been postulated as the pathogenic mecha- 


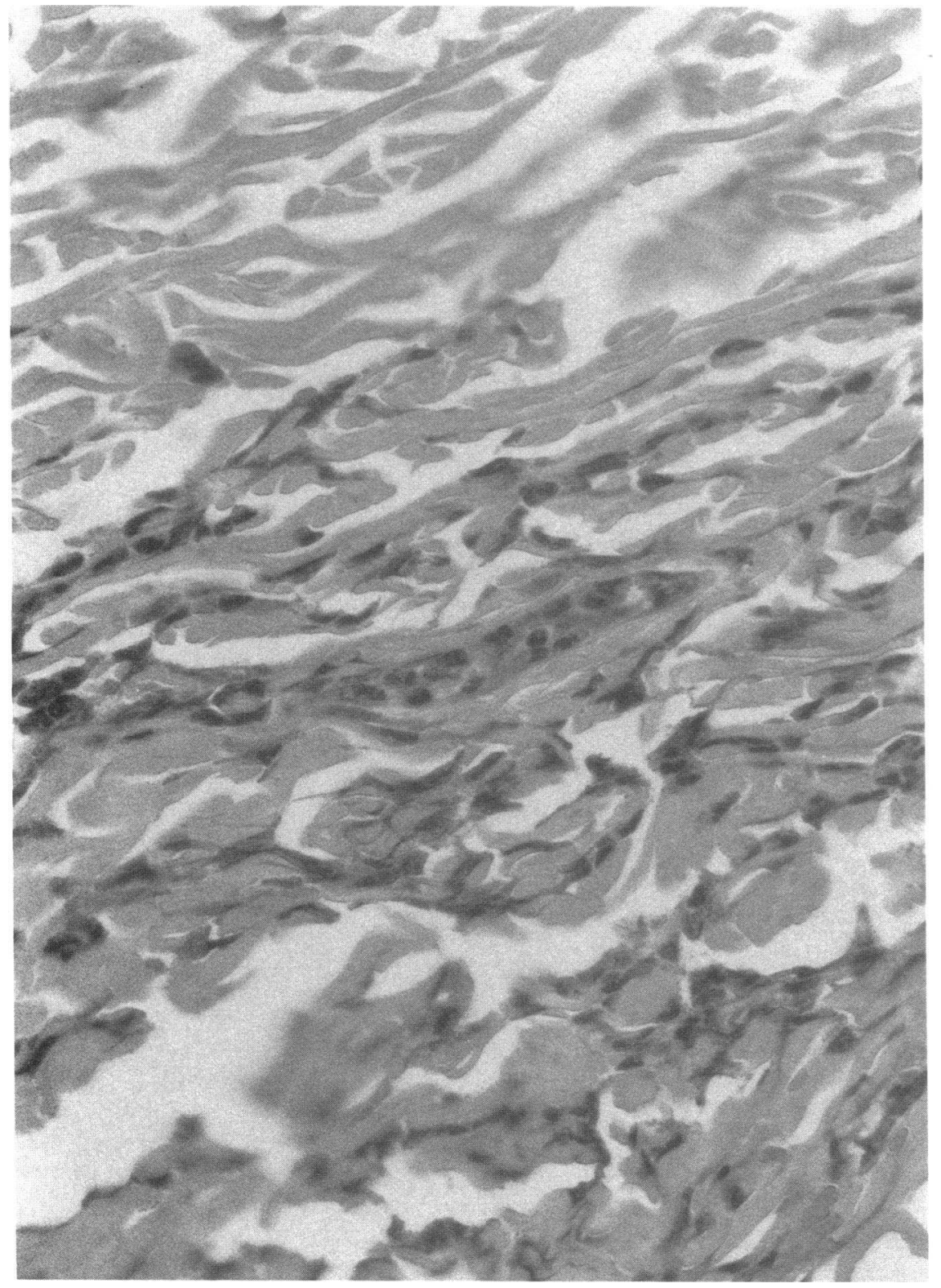

Figure 10. Histology of rabbit skin $20 \mathrm{~h}$ after the intradermal injection of C5a (100 $\mu$ l of zymosan-activated rabbit plasma). A marked dermal infiltrate of leukocytes has occurred, appearing similar in magnitude to that elicited by $30 \mu \mathrm{g}$ LPS (Fig. $1 c$ ),$\times 300$. nism of disseminated intravascular coagulation (32). Hopkins et al. (33) have described intravascular leukocyte aggregation in association with increased circulating levels of complement split products in patients with SLE; leukocyte aggregates in cerebral and mesenteric vessels were associated with cerebral and bowel infarction. In another study of SLE patients, a direct correlation between neutrophil Mac-1 expression and clinical disease severity was reported, with Mac-1 levels returning to normal as the disease improved (34). Upregulation of ICAM-1 has been demonstrated on both vascular endothelium and proximal tubule epithelium in murine autoimmune lupus nephritis, and is coincident with increased levels of IL-1 and TNF in the kidney (35).

Whereas the Arthus reaction has often been considered as an animal model representative of immune complex-me- diated vascular damage, the Shwartzman reaction may also have a correlate in human disease where vascular injury occurs without immune complex deposition. In referring to vasculitic syndromes, Fauci recently summarized "circulating immune complexes need not result in deposition of the complexes in blood vessels with ensuing vasculitis, and many patients with active vasculitis do not have demonstrable circulating or deposited immune complexes" (36). While cytokine priming (that is, stimulation of ICAM-1 and probably other endothelial cell adhesion molecules) may serve to direct leukocyte accumulation to infected tissues, sequential endothelial cell activation followed by activation of circulating leukocytes may lead to severe tissue damage. Further understanding of the role of adhesion events may provide ways to intervene in life-threatening, leukocyte-mediated inflammatory events. 

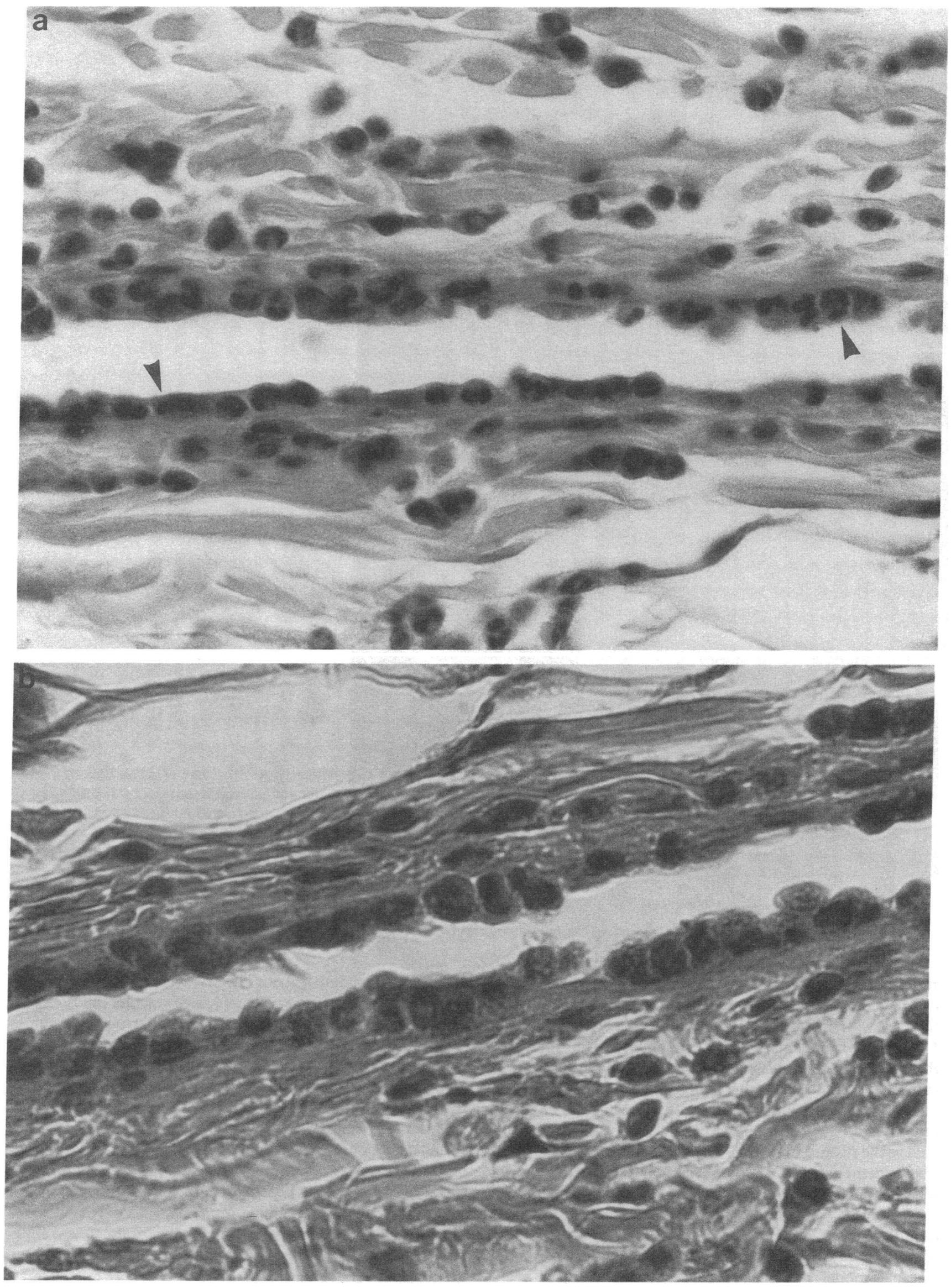

Figure 11. Histology of 6-h old Shwartzman lesions in rabbit skin, showing the effect of administration of antiCD11b, $2 \mathrm{mg} / \mathrm{kg}$, just before i.v. zymosan challenge. CD18/ICAM-1 dependent leukocyte-endothelial adhesion has not been completely inhibited as indicated by a single layer of leukocytes adherent to the vessel surface (arrow), but no leukocyte aggregation was seen in these skin sections. $(a) \times 400 ;(b)$ from another animal, $\times 600$. 


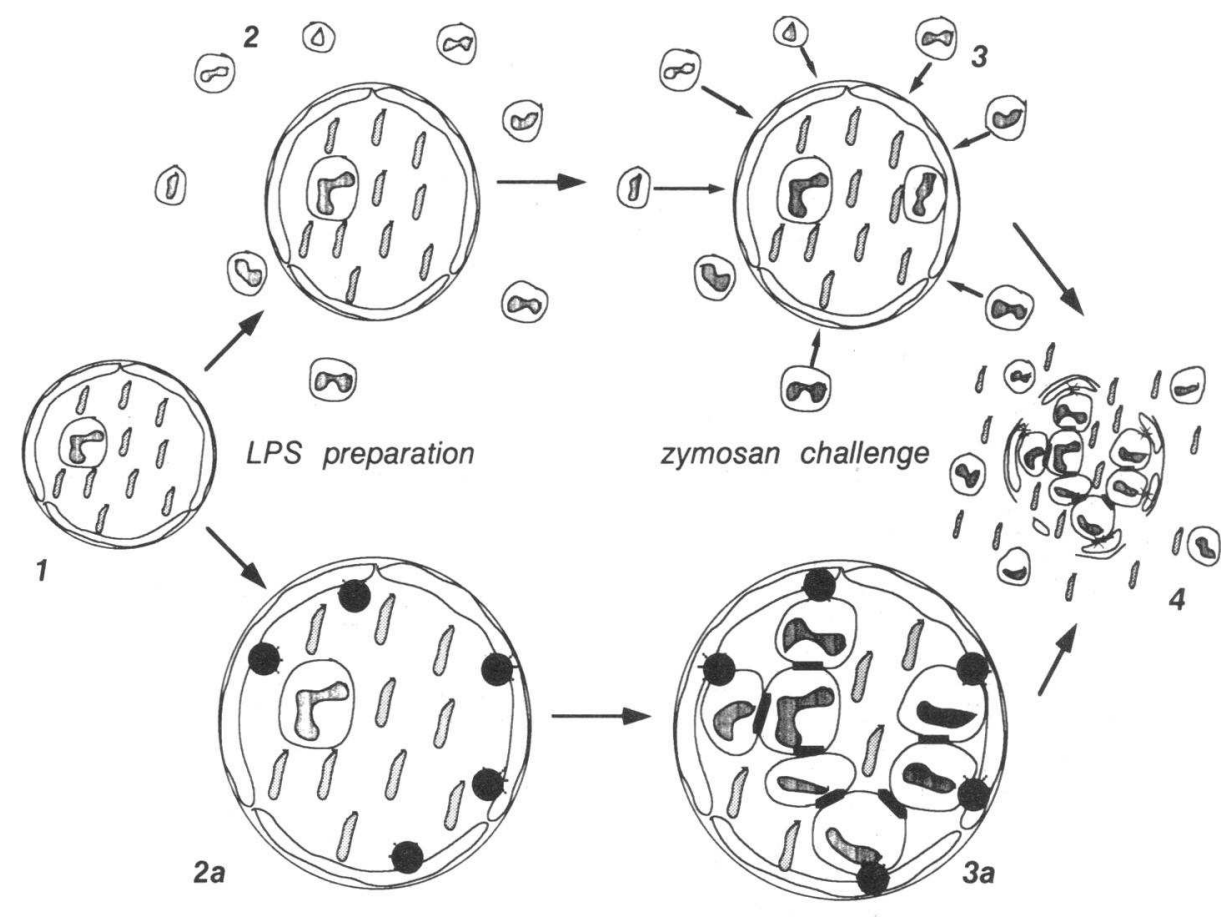

Figure 12. Proposed mechanism of the development of Shwartzman lesions. The most accepted pathway from normal morphology, vessel 1 , to hemorrhagic necrosis, vessel 4 , involves the accumulation of extravascular leukocytes, vessel 2 , in response to an agent capable of preparing the tissue for the Shwartzman response, in this case LPS. The challenge agent then leads to protease release from those leukocytes, vessel 3, thereby damaging the vessel and thus producing a hemorrhagic event. We propose an alternate mechanism. The critical effect of the preparative agent is the induction of increased ICAM-1 expression ( $\bullet$ ) on the vascular endothelium, vessel $2 \mathrm{a}$. The challenge agent then activates circulating leukocytes, causing rapid expression of the CD18 complex which binds to vessels now expressing ICAM-1, vessel 3a. In addition to CD18/ICAM-1 leukocyte-endothelial adhesion, a leukocyte-leukocyte aggregation also appears to be required $(\square)$. This intravascular adhesion/aggregation of leukocytes damages the vessel wall, resulting in hemorrhagic necrosis.

\section{Acknowledgments}

The authors gratefully acknowledge the technical assistance of Ronald Mosiello and Deborah Appley. We also thank Dr. Robert Rothlein for providing monoclonal antibodies, and Dr. Christina Myers for evaluating C5a effects on ICAM-1 expression in endothelial cell culture. We are also indebted to Gwyneth Van for providing expert immunohistochemical support. Thanks also to Carol Stearns for performing flow cytometry.

\section{References}

1. Springer, T. A. 1990. Adhesion receptors of the immune system. Nature (Lond.). 346:425-434.

2. Todd, R. F., M. A. Aranout, R. E. Rosin, C. A. Crowley, W. A. Peters, and B. M. Babior. 1984. Subcellular localization of the large subunit of Mol, a surface glycoprotein associated with neutrophil adhesion. J. Clin. Invest. 74:1280-1290.

3. Miller, L. J., D. F. Bainton, N. Borregaard, and T. A. Springer. 1987. Stimulated mobilization of monocyte Mac-1 and p150,95 adhesion proteins from an intracellular vesicular compartment to the cell surface. J. Clin. Invest. $80: 535-544$

4. Kishimoto, T. K., M. A. Jutila, E. L. Berg, and E. C. Butcher. 1989. Neutrophil Mac-1 and Mel-14 adhesion proteins inversely regulated by chemotactic factors. Science (Wash. DC). 245:1238-1241.

5. Buyon, J. P., S. B. Abramson, M. R. Philips, S. G. Slade, G. D. Ross, G. Weissmann, and R. J. Winchester. 1988. Dissociation between increased surface expression of gp165,95 and homotypic neutrophil aggregation. J. Immunol. 140:3156-3160.

6. Vedder, N. B., and J. M. Harlan. 1988. Increased surface expression of $\mathrm{CD} 1 \mathrm{lb} / \mathrm{CD} 18$ (Mac-1) is not required for stimulated neutrophil adherence to cultured endothelium. J. Clin. Invest. 81:676-682.

7. Smith, C. W., S. D. Marlin, R. Rothlein, C. Toman, and D. C. Anderson. 1989. Cooperative interactions of LFA-1 and Mac-1 with intercellular adhesion molecule-1 in facilitating adherence and transendothelial migration of human neutrophils in vitro. J. Clin. Invest. 83:2008-2017.
8. Pober, J. S., M. A. Gimbrone, Jr., L. A. Lapierre, D. L. Mendrick, W. Fiers, R. Rothlein, and T. A. Springer. 1986. Overlapping patterns of activation of human endothelial cells by interleukin-1, tumor necrosis factor and immune interferon. J. Immunol. 137:1893-1896.

9. Pohlman, T. H., K. A. Stanness, P. G. Beatty, H. D. Ochs, and J. M. Harlan. 1986. An endothelial cell surface factor induced in vitro by lipopolysaccharide, interleukin-1, and tumor necrosis factor increases neutrophil adherence by a CDw18-dependent mechanism. J. Immunol. 136:4548-4553.

10. Anderson, D. C., F. C. Schmalsteig, M. A. Aranout, S. Kohl, M. F. Tosi, N. Dana, G. J. Buffone, B. J. Hughes, B. R. Brinkley, W. D. Dickey, et al. 1984 Abnormalities of polymorphonuclear leukocyte function associated with a heritable deficiency of high molecular weight surface glycoproteins (GP138): a common relationship to diminished cell adherence. J. Clin. Invest. 74:536-551.

11. Kishimoto, T. K., N. Hollander, T. M. Roberts, D. C. Anderson, and T. A. Springer. 1987. Heterogenous mutations in the beta subunit common to the LFA-1, Mac-1, and p150,95 glycoproteins cause leukocyte adhesion deficiency. Cell. 50:193-202.

12. Philips, M. R., S. B. Abramson, and G. Weissmann. 1989. Neutrophil adhesion and autoimmune vascular injury. Clin. Aspects Autoimmun. 3:6-15.

13. Shwartzman, G. 1928. A new phenomenon of local skin reactivity to $B$. typhosus culture filtrate. Proc. Soc. Exp. Biol. Med. 25:560-561.

14. Movat, H. Z., B. J. Jaynes, S. Wasi, K. W. Movat, and M. M. Kopaniak 1980. Quantitation of the development and progression of the local Shwartzman reaction. In Bacterial Endotoxins and Host Response. M. K. Agarwal, editor Elsevier/North-Holland, Amsterdam. 179-201.

15. Marlin, S. D., and T. A. Springer. 1987. Purified intercellular adhesion molecule-1 (ICAM-1) is a ligand for lymphocyte-function-associated antigen 1 (LFA-1). Cell. 51:813-819.

16. Rothlein, R., and T. A. Springer. 1986. The requirement for lymphocytefunction-associated antigen 1 in homotypic leukocyte adhesion stimulated by phorbol ester. J. Exp. Med. 163:1132-1149.

17. Marlin, S. D., C. C. Morton, D. C. Anderson, and T. A. Springer. 1986 LFA-1 deficiency disease. Definition of the genetic defect and chromosomal mapping of alpha and beta subunits by complementation in hybrid cells. J. Exp. Med. 164:855-867.

18. Anderson, D. C., L. J. Miller, R. Schmalstieg, R. Rothlein, and T. A. Springer. 1986. Contributions of the Mac-1 glycoprotein family to adherence-dependent granulocyte functions: structure-function assessments employing subunit-specific monoclonal antibodies. J. Immunol. 137:15-27. 
19. Gu, J., and L. B. McGrath. 1990. Localized endocrine conversion of ventricular cardiocytes in ventricular aneurism. J. Histochem. Cytochem. 38:1659-1668.

20. Beck, G., G. S. Habicht, J. L. Benach, and F. Miller. 1986. Interleukin-1: a common endogenous mediator of inflammation and the local Shwartzman reaction. J. Immunol. 136:3025-3031.

21. Stetson, C. A., and R. A. Good. 1951. Shwartzman phenomenon-participation of polymorphonuclear leukocytes. J. Exp. Med. 93:49-53.

22. Movat, H. Z., and S. Wasi. 1985 . Severe microvascular injury induced by lysosomal releasates of human polymorphonuclear leukocytes. Am. J. Pathol. 121:404-417.

23. Halpern, B. N. 1963. Inhibition of the local hemorrhagic Shwartzman reaction by a polypeptide possessing potent antiprotease activity. Proc. Soc. Exp. Biol. Med. 115:273-276.

24. Movat, H. Z., C. E. Burrows, M. I. Cybulsky, and C. A. Dinarello. 1987. Acute inflammation and a Shwartzman reaction induced by interleukin-1 and tumor necrosis factor. Am. J. Pathol. 129:463-476.

25. Dustin, M. L., R. Rothlein, A. K. Bhan, C. A. Dinarello, and T. A Springer. 1986. Induction by IL-1 and interferon-gamma: tissue distribution, biochemistry, and function of a natural adhesion molecule (ICAM-1). J. Immunol. 137:245-254.

26. Fehr, J., C. Dahinden, and R. Russi. 1984. Formylated chemotactic peptides can mimic the secondary, provoking endotoxin injection in the generalized Shwartzman reaction. J. Infect. Dis. 150:160-161.

27. Rampart, M., and T. J. Williams. 1988. Evidence that neutrophil accumulation induced by interleukin-1 requires both local protein biosynthesis and neutrophil CD18 antigen expression in vivo. Br. J. Pharmacol. 94:1143-1148.

28. Argenbright, L. W., L. G. Letts, and R. Rothlein. 1991. Monoclonal antibodies to the leukocyte membrane CD18 glycoprotein complex and to intercellular adhesion molecule-1 inhibit leukocyte-endothelial adhesion in rabbits. $J$. Leukocyte Biol. 49:253-257.

29. Hammerschmidt, D. E., L. J. Weaver, P. Craddock, and H. Jacob. 1980. Association of complement activation and elevated plasma C5a with adult respiratory distress syndrome. Lancet. i:947-949.

30. Perez, H. D., J. K. Horn, and R. Ong. 1983. Complement (C5a)-derived chemotactic activity in serum from patients with pancreatitis. J. Lab. Clin. Med. 101:123-129.

31. Pinckard, R. N., M. S. Olson, P. C. Giclas, R. Terry, J. Boyer, and R. A O'Rourke. 1975. Consumption of classical complement components by heart subcellular membranes in vitro and in patients after acute myocardial infarction. J. Clin. Invest. 56:740-750.

32. Mori, W. 1983. Intravascular coagulation as a clinical manifestation of the Shwartzman reaction. Bibl. Haematol. 49:41-48.

33. Hopkins, P. T., H. M. Belmont, J. Buyon, M. Philips, G. Weissmann, and S. B. Abramson. 1988. Increased levels of plasma anaphlatoxins in systemic lupus erythematosus predicts flares of the disease and may elicit vascular injury in lupus cerebritis. Arthritis Rheum. 31:632-641.

34. Buyon, J. P., N. Shadick, and R. Berkman. 1988. Surface expression of gp165,95, the complement receptor CR3, as a marker of disease activity in systemic lupus erythematosus. Clin. Immunol. Immunopathol. 46:141-149.

35. Wuthrich, R. P., A. M. Jevnikar, F. Takei, L. H. Glimcher, and V. E Kelley. 1990. Intercellular adhesion molecule-1 (ICAM-1) expression is upregulated in autoimmune murine lupus nephritis. Am. J. Pathol. 136:441-450.

36. Fauci, A. S. 1987. The vasculitic syndromes. In Principles of Internal Medicine. E. Braunwald, K. Isselbacher, R. Petersdorf, J. Wilson, J. Martin, and A. Fauci, editors. McGraw-Hill, Inc., New York. 1438-1445. 